\title{
Molecular characterization of firework-related urban aerosols using Fourier transform ion cyclotron resonance mass spectrometry
}

Qiaorong Xie et al.

Correspondence to: Pingqing Fu (fupingqing @ tju.edu.cn)

The copyright of individual parts of the supplement might differ from the CC BY 4.0 License. 
Table S1. Number of compounds in each subgroup and arithmetic and weighted mean elemental ratio for each subgroup in LNY D and LNY N samples.

\begin{tabular}{|c|c|c|c|c|c|}
\hline & & All & $\mathrm{CHO}$ & $\mathrm{CHNO}$ & $\mathrm{CHOS}$ \\
\hline \multirow[t]{12}{*}{ LNY D } & Number frequency & 9511 & 3120 & 3604 & 1249 \\
\hline & Molecular weight (Da) & $448 \pm 97$ & $456 \pm 120$ & $472 \pm 112$ & $402 \pm 82$ \\
\hline & $\mathrm{O} / \mathrm{C}$ & $0.35 \pm 0.14$ & $0.31 \pm 0.12$ & $0.33 \pm 0.11$ & $0.40 \pm 0.13$ \\
\hline & $\mathrm{O} / \mathrm{C}_{\mathrm{w}}$ & 0.36 & 0.31 & 0.33 & 0.39 \\
\hline & $\mathrm{H} / \mathrm{C}$ & $1.18 \pm 0.36$ & $1.14 \pm 0.37$ & $1.08 \pm 0.29$ & $1.37 \pm 0.43$ \\
\hline & $\mathrm{H} / \mathrm{C}_{\mathrm{w}}$ & 1.18 & 1.10 & 1.05 & 1.46 \\
\hline & $\mathrm{OM} / \mathrm{OC}$ & $1.65 \pm 0.22$ & $1.50 \pm 0.17$ & $1.60 \pm 0.16$ & $1.80 \pm 0.21$ \\
\hline & $\mathrm{OM} / \mathrm{OC}_{\mathrm{w}}$ & 1.66 & 1.50 & 1.60 & 1.79 \\
\hline & DBE & $11.2 \pm 4.98$ & $12.2 \pm 5.95$ & $13.2 \pm 4.97$ & $7.21 \pm 4.65$ \\
\hline & $\mathrm{DBE}_{\mathrm{w}}$ & 10.8 & 11.8 & 13.1 & 6.05 \\
\hline & $\mathrm{DBE} / \mathrm{C}$ & $0.47 \pm 0.17$ & $0.47 \pm 0.18$ & $0.53 \pm 0.14$ & $0.37 \pm 0.21$ \\
\hline & $\mathrm{DBE} / \mathrm{C}_{\mathrm{w}}$ & 0.48 & 0.50 & 0.55 & 0.33 \\
\hline \multirow[t]{12}{*}{ LNY N } & Number frequency & 8426 & 2618 & 2515 & 1626 \\
\hline & Molecular weight (Da) & $413 \pm 85$ & $420 \pm 100$ & $415 \pm 86$ & $402 \pm 78$ \\
\hline & $\mathrm{O} / \mathrm{C}$ & $0.34 \pm 0.13$ & $0.28 \pm 0.12$ & $0.34 \pm 0.11$ & $0.34 \pm 0.16$ \\
\hline & $\mathrm{O} / \mathrm{C}_{\mathrm{w}}$ & 0.35 & 0.28 & 0.34 & 0.31 \\
\hline & $\mathrm{H} / \mathrm{C}$ & $1.28 \pm 0.38$ & $1.24 \pm 0.40$ & $1.14 \pm 0.34$ & $1.42 \pm 0.42$ \\
\hline & $\mathrm{H} / \mathrm{C}_{\mathrm{w}}$ & 1.28 & 1.24 & 1.08 & 1.50 \\
\hline & $\mathrm{OM} / \mathrm{OC}$ & $1.66 \pm 0.20$ & $1.47 \pm 0.16$ & $1.61 \pm 0.17$ & $1.71 \pm 0.25$ \\
\hline & $\mathrm{OM} / \mathrm{OC}_{\mathrm{w}}$ & 1.67 & 1.48 & 1.61 & 1.68 \\
\hline & DBE & $9.19 \pm 4.8$ & $9.98 \pm 5.23$ & $11.0 \pm 4.50$ & $6.80 \pm 4.30$ \\
\hline & $\mathrm{DBE}_{\mathrm{w}}$ & 8.71 & 9.38 & 11.3 & 5.81 \\
\hline & DBE/C & $0.43 \pm 0.20$ & $0.42 \pm 0.20$ & $0.51 \pm 0.17$ & $0.34 \pm 0.21$ \\
\hline & $\mathrm{DBE} / \mathrm{C}_{\mathrm{w}}$ & 0.43 & 0.43 & 0.54 & 0.31 \\
\hline
\end{tabular}


Table S2. Number of compounds in each subgroup and arithmetic and weighted mean elemental ratio for each subgroup in Normal D and Normal N samples.

\begin{tabular}{|c|c|c|c|c|c|}
\hline & & All & $\mathrm{CHO}$ & $\mathrm{CHNO}$ & $\mathrm{CHOS}$ \\
\hline \multirow[t]{12}{*}{ Normal D } & Number frequency & 5945 & 2168 & 2378 & 1399 \\
\hline & Molecular weight (Da) & $405 \pm 80$ & $400 \pm 90$ & $406 \pm 79$ & $405 \pm 83$ \\
\hline & $\mathrm{O} / \mathrm{C}$ & $0.36 \pm 0.15$ & $0.31 \pm 0.12$ & $0.34 \pm 0.10$ & $0.38 \pm 0.15$ \\
\hline & $\mathrm{O} / \mathrm{C}_{\mathrm{w}}$ & 0.36 & 0.31 & 0.34 & 0.36 \\
\hline & $\mathrm{H} / \mathrm{C}$ & $1.26 \pm 0.38$ & $1.14 \pm 0.38$ & $1.10 \pm 0.34$ & $1.44 \pm 0.40$ \\
\hline & $\mathrm{H} / \mathrm{C}_{\mathrm{w}}$ & 1.29 & 1.12 & 1.11 & 1.54 \\
\hline & $\mathrm{OM} / \mathrm{OC}$ & $1.69 \pm 0.22$ & $1.51 \pm 0.15$ & $1.62 \pm 0.16$ & $1.77 \pm 0.24$ \\
\hline & $\mathrm{OM} / \mathrm{OC}_{\mathrm{w}}$ & 1.69 & 1.51 & 1.62 & 1.76 \\
\hline & DBE & $9.01 \pm 4.25$ & $10.6 \pm 4.95$ & $11.0 \pm 4.26$ & $6.49 \pm 4.06$ \\
\hline & $\mathrm{DBE}_{\mathrm{w}}$ & 8.59 & 10.6 & 10.9 & 5.38 \\
\hline & DBE/C & $0.44 \pm 0.19$ & $0.47 \pm 0.19$ & $0.53 \pm 0.17$ & $0.34 \pm 0.20$ \\
\hline & $\mathrm{DBE} / \mathrm{C}_{\mathrm{w}}$ & 0.42 & 0.49 & 0.52 & 0.29 \\
\hline \multirow[t]{12}{*}{ Normal N } & Number frequency & 5454 & 2071 & 2140 & 1243 \\
\hline & Molecular weight (Da) & $416 \pm 88$ & $408 \pm 99$ & $414 \pm 89$ & $395 \pm 81$ \\
\hline & $\mathrm{O} / \mathrm{C}$ & $0.37 \pm 0.14$ & $0.31 \pm 0.11$ & $0.34 \pm 0.11$ & $0.41 \pm 0.14$ \\
\hline & $\mathrm{O} / \mathrm{C}_{\mathrm{w}}$ & 0.38 & 0.31 & 0.34 & 0.41 \\
\hline & $\mathrm{H} / \mathrm{C}$ & $1.24 \pm 0.36$ & $1.19 \pm 0.37$ & $1.11 \pm 0.30$ & $1.36 \pm 0.42$ \\
\hline & $\mathrm{H} / \mathrm{C}_{\mathrm{w}}$ & 1.23 & 1.16 & 1.09 & 1.40 \\
\hline & $\mathrm{OM} / \mathrm{OC}$ & $1.70 \pm 0.19$ & $1.51 \pm 0.15$ & $1.61 \pm 0.17$ & $1.81 \pm 0.22$ \\
\hline & $\mathrm{OM} / \mathrm{OC}_{\mathrm{w}}$ & 1.72 & 1.51 & 1.61 & 1.82 \\
\hline & DBE & $9.71 \pm 4.47$ & $10.2 \pm 4.77$ & $11.6 \pm 4.20$ & $7.17 \pm 4.48$ \\
\hline & $\mathrm{DBE}_{\mathrm{w}}$ & 9.20 & 10.0 & 11.4 & 6.49 \\
\hline & DBE/C & $0.46 \pm 0.18$ & $0.45 \pm 0.18$ & $0.52 \pm 0.15$ & $0.38 \pm 0.20$ \\
\hline & $\mathrm{DBE} / \mathrm{C}_{\mathrm{w}}$ & 0.46 & 0.47 & 0.53 & 0.36 \\
\hline
\end{tabular}


Table S3. Comparison of chemical characterization of water-soluble organic compounds in aerosol samples.

\begin{tabular}{|c|c|c|c|c|c|c|}
\hline Sampling site & Compounds & $\mathrm{O} / \mathrm{C}$ & $\mathrm{H} / \mathrm{C}$ & DBE & $\mathrm{DBE} / \mathrm{C}$ & Ref. \\
\hline Non-firework & All & $0.37 \pm 0.14$ & $1.24 \pm 0.37$ & $9.36 \pm 4.42$ & $0.45 \pm 0.18$ & This study \\
\hline Firework & All & $0.37 \pm 0.13$ & $1.23 \pm 0.37$ & $10.1 \pm 4.82$ & $0.45 \pm 0.18$ & This study \\
\hline Free tropospheric & All & $0.53 \pm 0.2$ & $1.48 \pm 0.3$ & $6.18 \pm 3.0$ & NA & (Mazzoleni et al., 2012) \\
\hline Free tropospheric & All & $0.46 \pm 0.13$ & $1.17 \pm 0.26$ & $10.7 \pm 4.0$ & $0.47 \pm 0.14$ & (Dzepina et al., 2015) \\
\hline Rural & All & $0.46 \pm 0.23$ & $1.34 \pm 0.39$ & $5.3 \pm 2.6$ & $0.45 \pm 0.21$ & (Lin et al., 2012) \\
\hline Rural & All & $0.28-0.32$ & $1.37-1.46$ & $6.30-7.45$ & $0.33-0.38$ & (Wozniak et al., 2008) \\
\hline Marin boundary layer & All & $0.36-0.42$ & $1.49-1.56$ & $5.88-6.76$ & $0.28-0.32$ & (Wozniak et al., 2014) \\
\hline Remote & All & $0.39-0.42$ & $1.30-1.34$ & $7.71-8.38$ & $0.41-0.42$ & (An et al., 2019) \\
\hline Free tropospheric & $\mathrm{CHO}$ & $0.47 \pm 0.2$ & $0.47 \pm 0.2$ & $0.47 \pm 0.2$ & NA & (Mazzoleni et al., 2012) \\
\hline Free tropospheric & $\mathrm{CHO}$ & $0.47 \pm 0.14$ & $1.19 \pm 0.27$ & $10.8 \pm 4.3$ & $0.46 \pm 0.14$ & (Dzepina et al., 2015) \\
\hline Rural & $\mathrm{CHO}$ & $0.40 \pm 0.21$ & $1.29 \pm 0.35$ & $5.6 \pm 2.4$ & $0.44 \pm 0.18$ & (Lin et al., 2012) \\
\hline Urban (hazy) & $\mathrm{CHO}$ & $0.41 \pm 0.19$ & $1.19 \pm 0.38$ & $8.0 \pm 3.9$ & $0.47 \pm 0.19$ & (Jiang et al., 2016) \\
\hline Free tropospheric & $\mathrm{CHNO}$ & $0.57 \pm 0.2$ & $0.57 \pm 0.2$ & $6.72 \pm 2$ & NA & (Mazzoleni et al., 2012) \\
\hline Free tropospheric & $\mathrm{CHNO}$ & $0.45 \pm 0.10$ & $1.14 \pm 0.22$ & $10.3 \pm 2.9$ & $0.51 \pm 0.12$ & (Dzepina et al., 2015) \\
\hline Rural & $\mathrm{CHNO}$ & $0.41 \pm 0.19$ & $1.15 \pm 0.31$ & $6.4 \pm 2.1$ & $0.59 \pm 0.16$ & (Lin et al., 2012) \\
\hline Urban (hazy) & $\mathrm{CHNO}$ & $0.45 \pm 0.22$ & $1.13 \pm 0.38$ & $8.8 \pm 4.0$ & $0.55 \pm 0.19$ & (Jiang et al., 2016) \\
\hline Free tropospheric & CHOS & $0.56 \pm 0.2$ & $1.64 \pm 0.3$ & $1.64 \pm 0.3$ & NA & (Mazzoleni et al., 2012) \\
\hline Free tropospheric & CHOS & $0.50 \pm 0.11$ & $1.75 \pm 0.31$ & $3.5 \pm 2.6$ & $0.2 \pm 0.14$ & (Dzepina et al., 2015) \\
\hline Rural & CHOS & $0.55 \pm 0.17$ & $1.67 \pm 0.31$ & $3.0 \pm 1.9$ & $0.25 \pm 0.16$ & (Lin et al., 2012) \\
\hline Urban (hazy) & $\mathrm{CHOS}$ & $0.65 \pm 0.28$ & $1.64 \pm 0.37$ & $3.4 \pm 2.4$ & $0.26 \pm 0.18$ & (Jiang et al., 2016) \\
\hline
\end{tabular}


Daytime
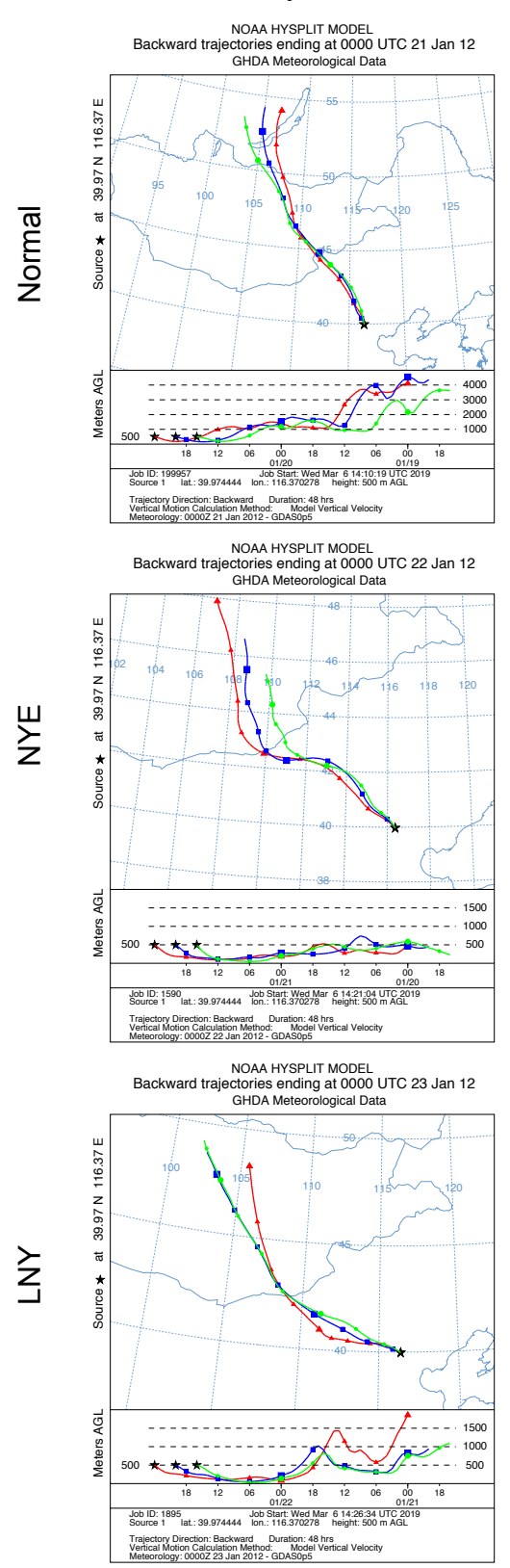

Nighttime

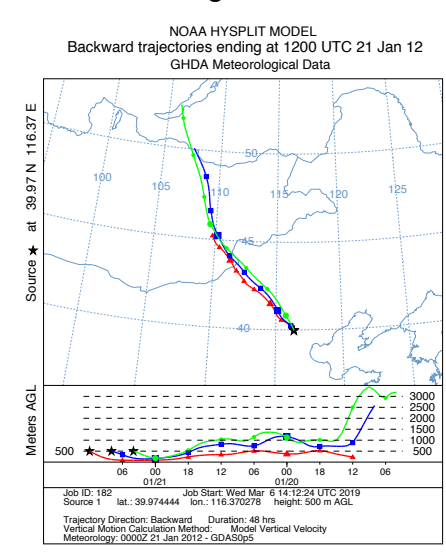

NOAA HYSPLIT MODEL
Backward trajectories ending at 1200 UTC 22 Jan 12

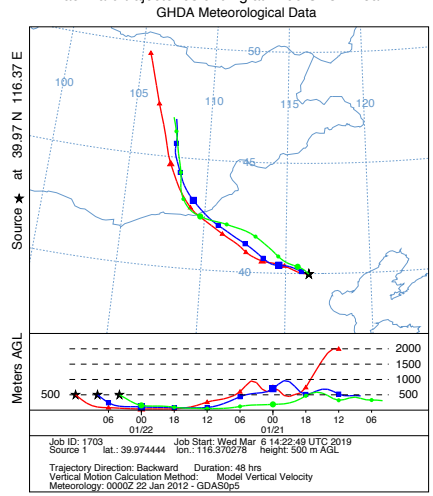

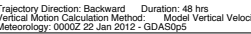
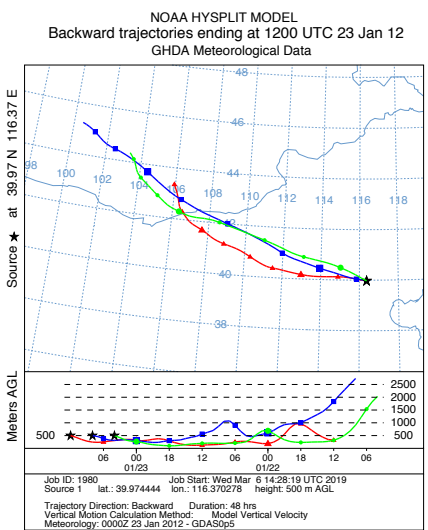

Figure S1: The clustering air mass two-day backward trajectories. 


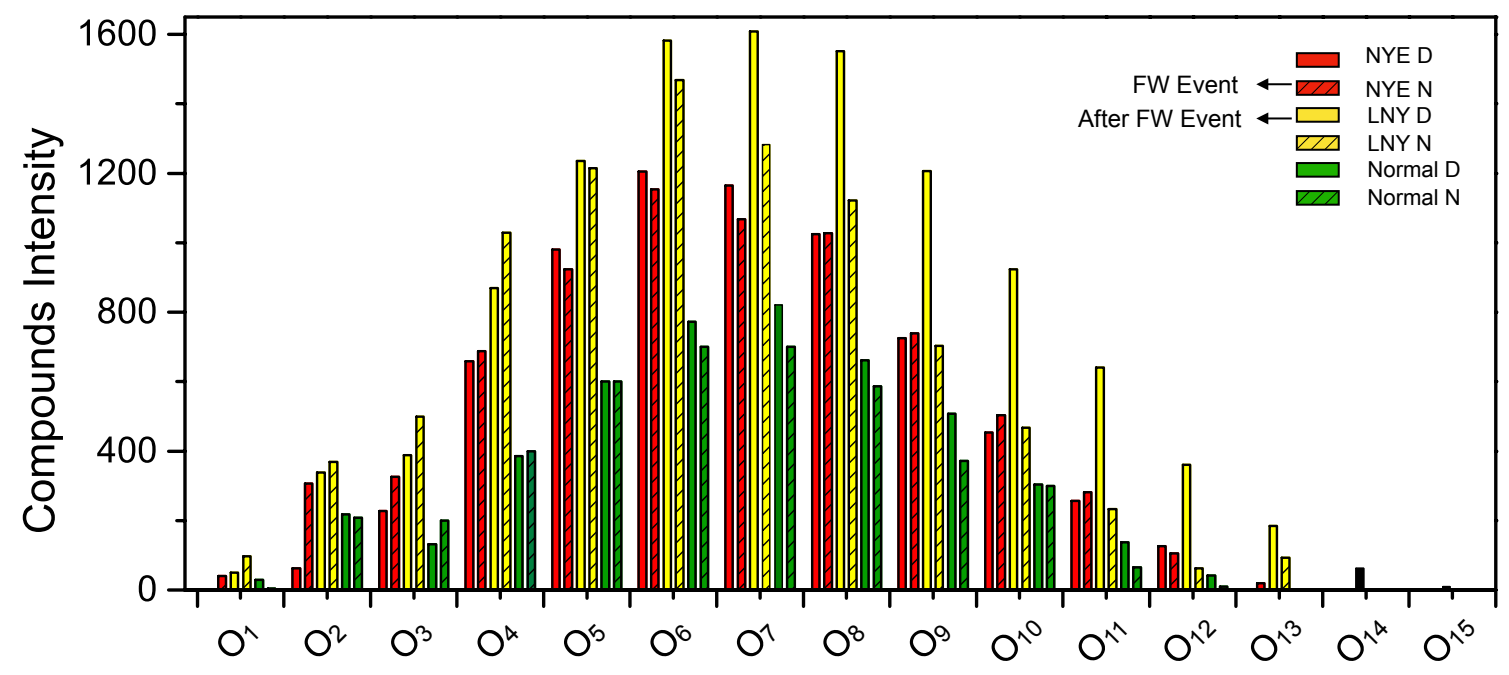

Figure S2: Intensity of CHO species of subgroups according to the number of $\mathrm{O}$ atoms in their molecules. 

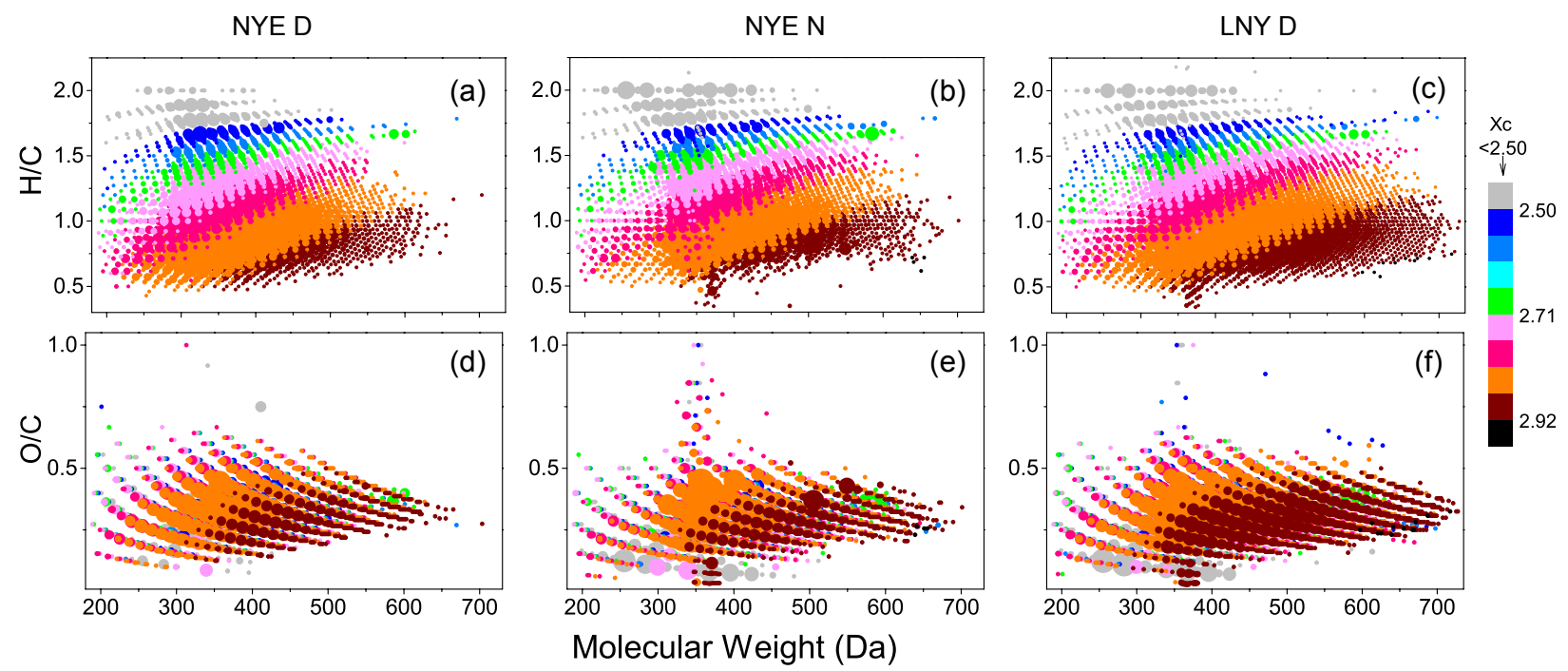

Figure S3: The $\mathrm{H} / \mathrm{C}(\mathbf{a}, \mathbf{b}, \mathbf{c})$ and $\mathrm{O} / \mathrm{C}(\mathbf{d}, \mathbf{e}, \mathbf{f})$ ratios of $\mathrm{CHO}$ formulae are shown as a function of their neutral mass from NYE D (a, d), NYE N (b, e) and LNY D $(\mathbf{c}, \mathbf{f})$ samples with their $\mathrm{X}_{\mathrm{c}}$ values color-coded. Grey data points indicate non-aromatic compounds $\left(\mathrm{X}_{\mathrm{c}}<2.5\right)$, blue to green data $\left(2.5<\mathrm{X}_{\mathrm{c}}<2.71\right)$ are mono-aromatic compounds and pink to black data $\left(\mathrm{X}_{\mathrm{c}}>2.71\right)$ includes PAHs. The size of the symbols reflects the relative peak intensities of molecular formulae on a logarithmic scale. 

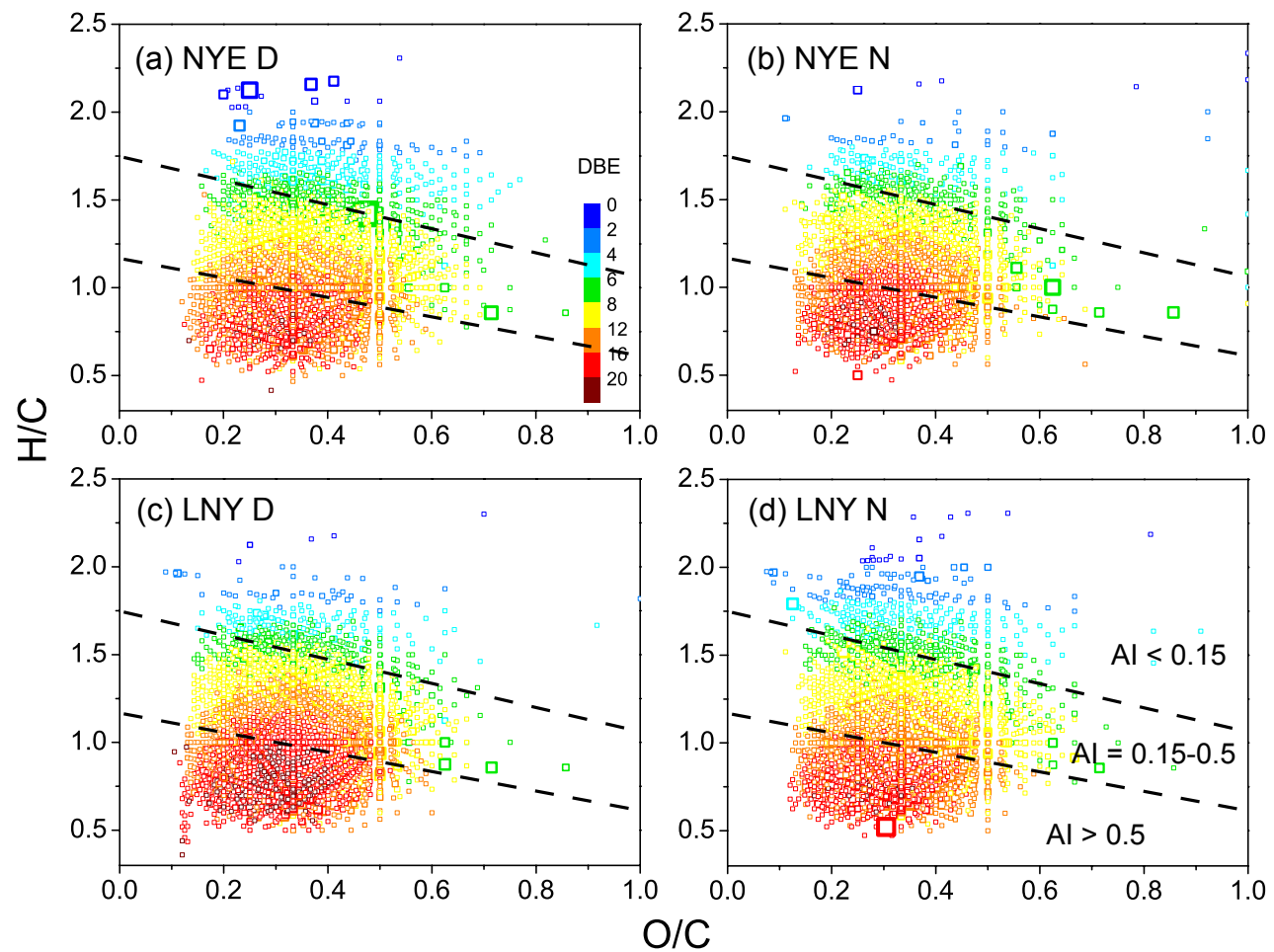

Figure S4: Van Krevelen diagrams (the $\mathrm{H} / \mathrm{C}$ via $\mathrm{O} / \mathrm{C}$ ratios) for the $\mathrm{CHNO}$ compounds with various aromatic index (AI) values ranges. The dashes lines separate the different AI regions. The size of the symbols reflects the relative peak intensities of compounds on a logarithmic scale. 


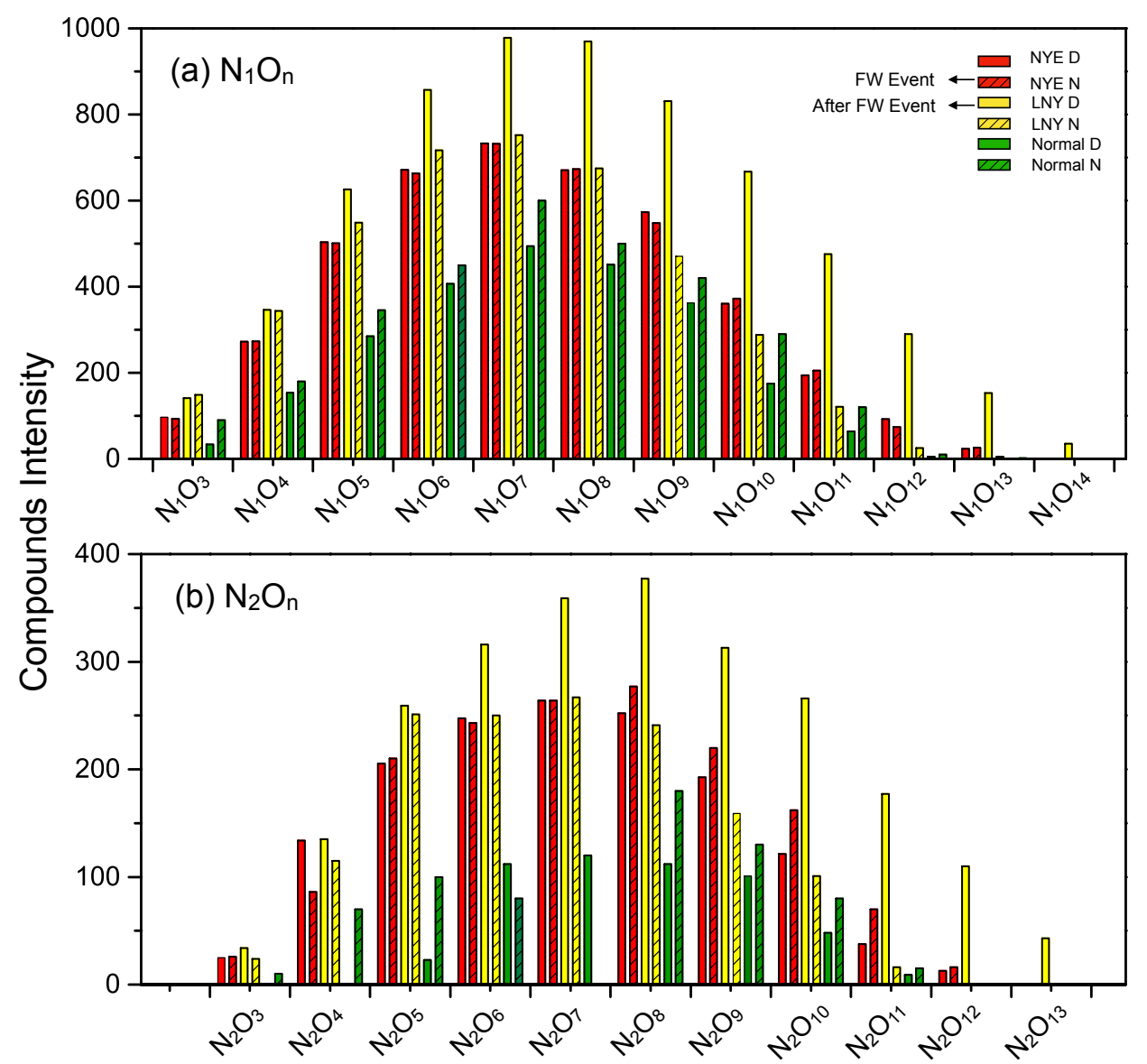

Figure S5: Intensity of CHNO species of subgroups according to the number of $\mathrm{N}$ and $\mathrm{O}$ atoms in their molecules. 


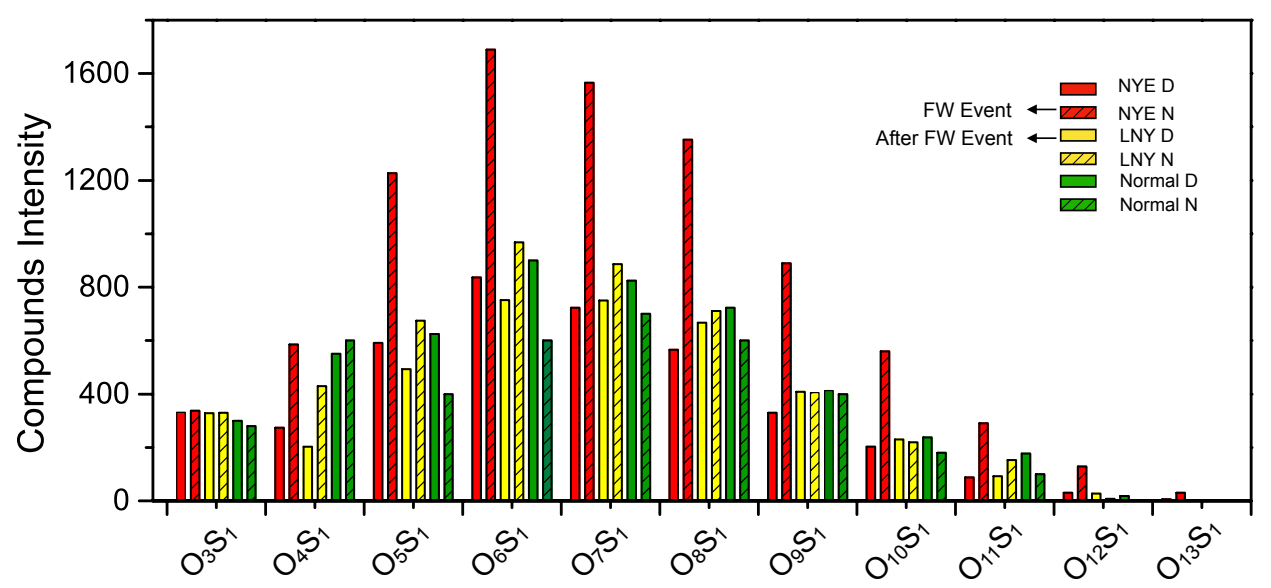

Figure S6: Intensity of CHOS species of subgroups according to the number of $\mathrm{O}$ and $\mathrm{S}$ atoms in their molecules. 

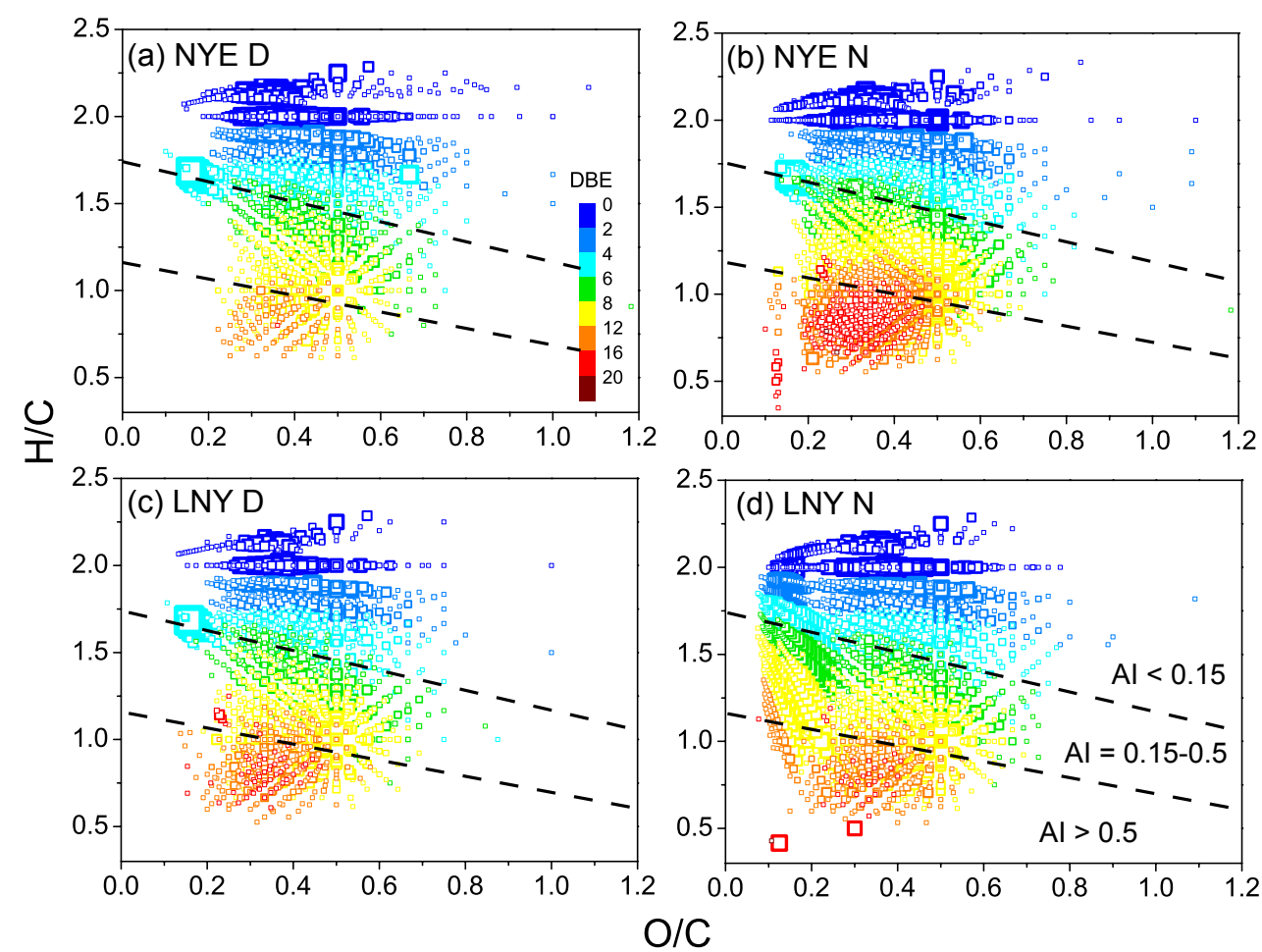

Figure S7: Van Krevelen diagrams (the $\mathrm{H} / \mathrm{C}$ via $\mathrm{O} / \mathrm{C}$ ratios) for the $\mathrm{CHOS}$ compounds with various aromatic index (AI) values ranges. The dashes lines separate the different AI regions. The size of the symbols reflects the relative peak intensities of compounds on a 5 logarithmic scale. 


\section{References}

An, Y. Q., Xu, J. Z., Feng, L., Zhang, X. H., Liu, Y. M., Kang, S. C., Jiang, B., and Liao, Y. H.: Molecular characterization of organic aerosol in the Himalayas: insight from ultra-high-resolution mass spectrometry, Atmos. Chem. Phys., 19, 1115-1128, 10.5194/acp-191115-2019, 2019.

5 Dzepina, K., Mazzoleni, C., Fialho, P., China, S., Zhang, B., Owen, R. C., Helmig, D., Hueber, J., Kumar, S., Perlinger, J. A., Kramer, L. J., Dziobak, M. P., Ampadu, M. T., Olsen, S., Wuebbles, D. J., and Mazzoleni, L. R.: Molecular characterization of free tropospheric aerosol collected at the Pico Mountain Observatory: a case study with a long-range transported biomass burning plume, Atmos. Chem. Phys., 15, 5047-5068, 10.5194/acp-15-5047-2015, 2015.

Jiang, B., Kuang, B. Y., Liang, Y., Zhang, J., Huang, X. H., Xu, C., Yu, J. Z., and Shi, Q.: Molecular composition of urban organic 10 aerosols on clear and hazy days in Beijing: a comparative study using FT-ICR MS, Environ. Chem., 13, 888-901, 2016.

Lin, P., Rincon, A. G., Kalberer, M., and Yu, J. Z.: Elemental composition of HULIS in the Pearl River Delta Region, China: Results inferred from positive and negative electrospray high resolution mass spectrometric data, Environ. Sci. Technol., 46, 7454-7462, 2012.

Mazzoleni, L. R., Saranjampour, P., Dalbec, M. M., Samburova, V., Hallar, A. G., Zielinska, B., Lowenthal, D. H., and Kohl, S.: Identification of water-soluble organic carbon in non-urban aerosols using ultrahigh-resolution FT-ICR mass spectrometry: Organic anions,

15 Environ. Chem., 9, 285-297, 2012.

Wozniak, A. S., Bauer, J. E., Sleighter, R. L., Dickhut, R. M., and Hatcher, P. G.: Technical Note: Molecular characterization of aerosolderived water soluble organic carbon using ultrahigh resolution electrospray ionization Fourier transform ion cyclotron resonance mass spectrometry, Atmos. Chem. Phys., 8, 5099-5111, 10.5194/acp-8-5099-2008, 2008.

Wozniak, A. S., Willoughby, A. S., Gurganus, S. C., and Hatcher, P. G.: Distinguishing molecular characteristics of aerosol water soluble organic matter from the 2011 trans-North Atlantic US GEOTRACES cruise, Atmos. Chem. Phys., 14, 8419-8434, 2014. 